\title{
Yod
}

Revue des études hébraïques et juives

19 | 2014

Aharon Appelfeld, cinquante ans d'écriture

\section{La symbolique des langues de la nature dans les nouvelles d'Aharon Appelfeld}

The Symbolism of Natural Languages in Aharon Appelfeld's Short Stories הסמליות בתיאורי הטבע בסיפורי אפלפלד

\section{Michèle Tauber}

\section{(Q) OpenEdition}

Journals

Édition électronique

URL : https://journals.openedition.org/yod/2100

DOI : 10.4000/yod. 2100

ISSN : 2261-0200

Éditeur

INALCO

Édition imprimée

Date de publication : 30 mai 2014

ISBN : 978-2-85831-214-6

ISSN : 0338-9316

Référence électronique

Michèle Tauber, "La symbolique des langues de la nature dans les nouvelles d'Aharon Appelfeld », Yod [En ligne], 19 | 2014, mis en ligne le 16 avril 2014, consulté le 08 juillet 2021. URL : http://

journals.openedition.org/yod/2100; DOI : https://doi.org/10.4000/yod.2100

Ce document a été généré automatiquement le 8 juillet 2021.

\section{(c) (1) 8)}

Yod est mis à disposition selon les termes de la Licence Creative Commons Attribution - Pas d'Utilisation Commerciale 4.0 International. 


\title{
La symbolique des langues de la nature dans les nouvelles d'Aharon Appelfeld
}

The Symbolism of Natural Languages in Aharon Appelfeld's Short Stories הסמליות בתיאורי הטבע בסיפורי אפלפלד

\author{
Michèle Tauber
}

1 Avant d'entamer un fructueux parcours de romancier, Aharon Appelfeld a commencé sa carrière en tant que nouvelliste. Entre 1962 et 1971, il a publié cinq recueils de nouvelles ${ }^{1}$ sans compter de multiples textes qui ont vu le jour dans des revues littéraires ${ }^{2}$.

2 C'est dans la nature et ses éléments multiples que le jeune Appelfeld puise les langages qui lui permettent de forger une symbolique où la polysémie n'a d'égal que les variations musicales de la langue hébraïque. Tour à tour, l'eau, la forêt, la steppe glacée ou la brûlure du soleil deviennent des moyens d'expression, de véritables langages où affleurent sensations, souvenirs et états d'âme. La nature incarne ainsi la muse inspiratrice d'une palette de nouveaux symboles créés par l'artiste.

\section{Les langages de l'eau}

3 L'élément liquide se présente sous deux formes : l'eau douce et la mer.

4 L'eau douce est tantôt évoquée comme une eau bienfaisante et amicale, tantôt comme le symbole du temps qui passe et de la brièveté de l'existence et par là même comme une force maléfique. Il arrive aussi que ces deux perspectives soient entrelacées et que la menace tapie dans l'une soit voilée par les charmes de l'autre.

5 Le fleuve et la rivière sont liés avant tout à des souvenirs d'enfance idylliques. Pour le personnage adulte en quête du paysage idéalisé de l'enfance, le fleuve représente un passage initiatique, une sorte de re-naissance lui ouvrant la voie, certes illusoire, du bonheur simple d'autrefois. Ainsi les deux personnages de Rencontre ${ }^{3}$ se remémorant 
leur bourgade natale alors qu'ils arpentent Jérusalem, évoquent la fascination qu'exerçait la rivière aux jours de leur enfance. Le fleuve incarne non seulement le lieu de l'enfance, mais il apparaît aussi comme une divinité qui se "révèle " aux yeux émerveillés de l'enfant. La sensualité qui imprègne cette vision se traduit par une sensation proprement physique de l'eau rafraîchissante sur le corps. Et c'est cette sensation que cherchent à retrouver bien des années plus tard les deux personnages originaires de Lishtshik, cette bourgade oubliée.

$6 \quad$ Mais l'eau douce représente aussi le monde des Autres, c'est-à-dire des non-Juifs. Comme telle, elle incarne une force généralement négative qui agit de diverses façons. Dans la nouvelle Rushka ${ }^{4}$, un pêcheur et sa femme, Juifs des campagnes vivant en retrait de la tradition, sont tous deux attirés par l'eau du fleuve qui va causer leur perte. Dès le printemps, la jeune femme passe toutes ses journées au bord du fleuve où elle se baigne l'été venu. Le fleuve joue dans ce récit le rôle d'un personnage à part entière : Rushka succombe à la séduction et à la fascination de l'eau et oublie ses origines et son passé. Le pêcheur quant à lui, doit affronter l'hiver et c'est là que le fleuve se révèle dans toute sa cruauté. La glace pétrifie l'eau en d'autres temps si accueillante. Le paysage entier est paralysé et tout s'immobilise : terrible concrétisation de l'oubli qui fige et raidit toute existence. Peu à peu la métaphore du souvenir gelé gagne le fleuve tout entier. L'eau, élément mouvant et ondoyant par excellence, est prise à son tour dans la rigidité glacée et emprisonne la barque du pêcheur, lui ôtant ainsi tout moyen de survie. Impitoyable est le langage du fleuve : le pêcheur comprend confusément que l'eau n'est pas la mère nourricière qu'il a servie avec dévotion. Loin d'être pour lui un refuge, elle lui montre au contraire qu'elle est aussi l'incarnation de la mort.

7 D'autres personnages connaissent cette fusion avec l'élément liquide, symbole de leur désir d'adéquation avec le monde non-juif qui les entoure. Ainsi dans la nouvelle $L a$ métamorphose ${ }^{5}$ l'homme et la femme se baignent tous deux dans le fleuve et nagent " avec aisance ». Le contact avec l'eau produit un effet immédiat : la femme rit «à la façon des paysannes pendant les journées de cueillette des plantations $»^{6}$. L'eau demeure ainsi au centre de l'inexorable processus d'assimilation à l'univers non-juif. Paolina, cousine du jeune narrateur de la longue nouvelle Comme la pupille de l'œil', est une violoncelliste de talent. Mais terrorisée par son professeur et étrangement fascinée par le milieu non-juif, elle abandonne peu à peu la musique, fraye avec un jeune paysan et subit, elle aussi, une métamorphose étonnante :

Comme Paolina a changé. Elle se promène pieds nus et se roule dans l'herbe. Elle a perdu son petit rire étouffé. À présent son rire nous effraie tous. Son front a bruni, et ses yeux brillent d'une joie qui nous est étrangère. Maman la supplie en vain : «Mais joue donc quelque chose.» Toute la journée dans le fleuve. Le soir elle s'endort tout habillée, comme une vulgaire servante ${ }^{8}$.

8 La jeune artiste raffinée adopte le comportement des jeunes paysans et change complètement de langage. Elle cesse de s'exprimer par le langage musical et utilise désormais le sens le plus animal, celui du toucher, pour fusionner avec la nature solide et liquide, l'herbe et le fleuve. Le "front bruni » et la "joie étrangère » accentuent l'aspect inquiétant de cette métamorphose qui éloigne de plus en plus la jeune fille de sa famille. À la fin de la description, Paolina a pris l'aspect d'une servante et semble avoir bel et bien perdu toute attache qui la reliait à ses origines. En contrepoint à ce personnage, apparait une toute jeune servante, Pissula, pour laquelle l'eau est une seconde nature: «Dans l'eau du fleuve, on la sentait libre et légère comme l'une des créatures de l'onde, tour à tour plongeant ou flottant à la surface. Elle remontait parfois 
du fond brandissant un poisson ou une écrevisse. ${ }^{9}$ Mais tandis que Pissula, non-juive, est réellement une fille de la nature qui fusionne avec le paysage - le narrateur la compare à un caméléon - Paolina et Rushka n'échappent pas à leur condition humaine juive.

9 Révélant sa véritable nature inconstante, l'eau apparait dans ces nouvelles comme un redoutable tartuffe qui séduit ses proies pour mieux les perdre. Autant les personnages de paysans chrétiens sont en symbiose spontanée avec l'élément liquide, autant les personnages juifs vivent cette tentative de fusion dans les plus grands tourments, car elle est simultanément synonyme de la rupture d'avec leur être le plus intime et à l'origine de l'oubli de leurs racines.

\section{La mer}

10 L'une des premières mentions de la mer apparait dans une nouvelle de 1963, Sur le rivage $^{10}$, où Appelfeld relate les attentes des rescapés cantonnés sur les côtes de l'Italie du Sud. Dès la première phrase, l'ironie est de mise : «Tout de suite après la guerre de grandes possibilités s'ouvrirent aux gens. Les trains affluaient vers les ports, vers les portails bleus ${ }^{11}$ que le monde venait d'ouvrir $»^{12}$. Mais dès la phrase suivante, "Quelques-uns réussirent à grimper à bord des bateaux, mais tous les autres restèrent là, sur le rivage, à côté des petites baraques... " ${ }^{13}$, le lecteur comprend que ce «bleu », symbole d'un avenir meilleur, n'est qu'un leurre et que la mer n'apportera pas le salut espéré.

11 La situation est identique pour les rescapés de Hanoucca $1946^{14}$. En plein hiver, dans une baraque, sur une plage italienne aux flots déchaînés, un fils naît aux Fridel. Le couple a réussi à survivre à la guerre en se cachant dans la forêt. Mais ces trois années ont laissé à Fridel le souvenir de l'horreur vécue, laquelle s'exprime dans son œil bleu étincelant, véritable incarnation de sa mémoire brûlante. La mer, elle, incarne l'inhumanité et l'absence de toute mémoire : «Des vents froids soufflaient du rivage. L'eau avait perdu sa couleur bleue et les lourdes vagues crachaient une écume verte $»^{15}$. Peu à peu, le «bleu » s'affaiblit chez Fridel de même que sa vie l'abandonne, et qu'il disparaît sans laisser de traces. En même temps que s'accomplit inexorablement la perte de la vue et de la vie chez Fridel père, la vision du nouveau-né Fridel se fait de plus en plus perçante jusqu'au moment où il apparaît qu'il a hérité de l'œil de son père et, partant, de toute la mémoire paternelle. La tempête s'est enfin calmée et de façon inattendue, la couleur bleue que l'on croyait disparue se manifeste timidement dans le ciel.

\section{Mer sacrée, mer sanctuaire}

12 La mer n'incarne pas seulement le mystère de l'oubli et du silence. Elle peut aussi se muer en sanctuaire, réceptacle métaphorique d'un sacré devenu profane et dans lequel les personnages tentent de trouver de nouveaux points de repère à leur existence.

13 Dans une telle perspective de purification, Tshukhovski, dans Aux îles Saint George ${ }^{16}$, prend le large après la guerre pour une île perdue au milieu de la Méditerranée. Il aspire à une rédemption en solitaire. La faute dont il s'accuse sans cesse n'est autre que le terrible sentiment de culpabilité éprouvé par le survivant dont toute la famille a été exterminée. 
14 La nouvelle s'ouvre sur une vision mythologique de l'expiation: un vieux pêcheur transporte Tshukhovsky dans sa barque jusqu'aux îles Saint George. L'image n'est pas sans rappeler, écrit Gila Ramras-Rauch ${ }^{17}$, la traversée du Styx lorsque les âmes des damnés sont transférées dans l'au-delà infernal à cause de leurs forfaits. Or le lieu choisi pour faire pénitence porte un nom de saint et, précise Lily Rattok ${ }^{18}$, l'allusion à la mise à mort du dragon par Saint-Georges n'est certes pas fortuite. Il s'agit pour le personnage d'anéantir tous les "dragons" du passé qui l'obsèdent. Mais ce faisant, Tshukhovsky, si désireux de rompre avec son existence antérieure, est saisi par la nostalgie de son enfance. Ainsi, " parallèlement au voyage en mer en direction de l'île, puis au voyage sur l'île à la découverte de ses paysages désolés, s'accomplit dans le personnage un voyage vers son passé $»^{19}$.

15 Entourant ce microcosme de la rédemption que sont les îles, la mer fait figure d'immense sanctuaire à la fois menaçant et protecteur. Tshukhovsky a besoin de sentir cette proximité de l'élément liquide qui le sépare d'un monde qu'il rejette et l'y rattache en même temps : c'est là qu'il peut oser affronter ce qu'il avait gardé enfoui au plus profond de lui-même, son passé d'avant-guerre.

16 La mer-sanctuaire ne serait-elle alors qu'un leurre, qu'une illusion pour ces personnages qui semblent tant compter sur son effet thérapeutique, purificateur, voire rédempteur? Ou bien aurait-elle une signification équivoque : d'une part l'incarnation d'un ultime réceptacle des dernières espérances, la sépulture des fragiles étincelles d'existence qui subsistent encore chez les survivants et d'autre part, le signe infime, la promesse tacite qu'elle peut à nouveau générer la vie?

\section{Les langages de la forêt}

17 Au langage de l'élément liquide et insaisissable répond comme en écho solide celui de la forêt et de ses arbres. Ces deux aspects du paysage sont d'autant plus complémentaires l'un de l'autre que la forêt dépend, pour sa survie, de la présence des eaux souterraines. Ainsi enchâssées l'une dans l'autre, l'eau et la forêt reflètent dans l'univers d'Appelfeld une surprenante gémellité. Celle-ci se traduit par l'aspect à la fois protecteur et menaçant de la forêt, capable, à l'instar de l'eau, d'offrir un havre de paix et un refuge sûr, puis de devenir au contraire le lieu de tous les périls. Elle est aussi un lieu de métamorphose pour les hommes où les fugitifs perdent leurs racines pour se fondre dans celles de la forêt et du monde étranger.

$18 \mathrm{Au}$ commencement était l'arbre. Dès le troisième jour de la création apparaît l'arbre fruitier, indispensable compagnon de l'homme, qui l'accompagne désormais tout au long du récit biblique. La nouvelle Dans le vallon fertile $e^{20}$ met en scène un survivant qui trouve refuge dans un vallon paradisiaque, du moins au premier abord. En effet, la description d'un paysage à la fin de l'été au moment de la cueillette des pommes juteuses et dorées évoque immédiatement le jardin d'Eden et ses arbres chargés de fruits succulents et tentateurs :

Ce fut la nuit et à nouveau le jour. Les arbres aspiraient en eux leur sève ultime, le soleil répandait sa chaleur et embrasait ses ors sur les pommes. [...] Jour et nuit la cueillette se poursuivait. L'abondance menaçait de submerger le vallon. Les fruits récoltés s'amoncelaient, mais les arbres courbaient toujours la tête, cherchant une main disponible ${ }^{21}$. 


\section{Les forêts de Pologne}

19 Il arrive aussi que la forêt demeure bel et bien vivante dans la mémoire de ceux qui l'ont aimée et soignée et qui, au-delà du temps et de l'éloignement, continuent à lui vouer un véritable culte. Ainsi le personnage de Scharfstein dans la nouvelle Lentement ${ }^{22}$ a emporté avec lui à Jérusalem son paysage intérieur, à savoir les forêts de Bialystok où il était jadis garde forestier. Son mode de vie, son comportement, sa façon de parler sont entièrement conditionnés par sa vie d'autrefois. Il va même jusqu'à identifier les rides d'un visage humain aux anneaux des ans d'un tronc fendu.

Scharfstein qui a non seulement perdu ses forêts, mais encore très sûrement tout ce qui s'y rattachait, parents, famille, maison, ne peut concevoir une suite à son existence sans les forêts de Bialystok en toile de fond imaginaire. De façon paradoxale le garde forestier est à la fois séparé du lieu de sa jeunesse et n'en a jamais été aussi proche. Tout son environnement s'est en quelque sorte transformé en forêt dans la mesure où les maisons, le quartier, la ville - en l'occurrence Jérusalem - ont adopté le rythme de croissance des arbres, c'est-à-dire la lenteur. La mémoire de Sharfstein est plus forte que le temps lui-même et réussit à transplanter tout un paysage de forêts à des annéeslumière de son lieu d'origine. Le rythme de vie du personnage est indéfectiblement lié à celui des arbres. "Les arbres poussent lentement» demeure son leitmotiv de prédilection. Ainsi lorsque les affaires ne sont pas très fructueuses, Scharfstein, reconverti en agent immobilier, commente la situation en des termes qui relèvent plutôt de la croissance des arbres ou d'un fleuve en crue :

Très lentement, les images défilent. On ne doit pas en ralentir le mouvement, mais on peut en suivre l'écoulement. Les maisons ne s'achètent pas, ne se vendent pas. "Les maisons sont soumises au pouvoir du temps", dit Scharfstein. "Un propriétaire doit attendre la période de maturité. " Très lentement se font les tractations, dans une sorte d'écoulement dense. Parfois c'est la morne routine, parfois l'explosion; puis à nouveau, comme dans un mouvement de réconciliation, les eaux rejoignent leur lit ${ }^{23}$.

\section{L'arbre-sanctuaire}

21 L'arbre incarne parfois, à lui seul, le symbole de toute une forêt. À ce titre, il recèle alors en lui la part de sacré ordinairement dévolue à une forêt entière. Ainsi l'arbre du Paradis créé pour offrir à l'homme toute une palette de sensations peut aussi incarner un lieu saint. Deux lieux bibliques au moins ont été témoins de grandes révélations divines. Il s'agit du chêne de Moreh, non loin de Sichem et de la chênaie de Mambré, proche de Hébron ${ }^{24}$. Dans ces deux passages, l'arbre fait office de sanctuaire sitôt après une révélation divine et, à deux reprises, Abram y bâtit un autel. Dans l'antiquité grecque, la ville de Dodone joue un rôle politique important grâce à son célèbre oracle de Zeus : les prêtres et les prêtresses y rendent la réponse des dieux en interprétant le bruissement du feuillage des chênes sacrés. Dans L'Odyssée, Ulysse vient consulter deux fois, sur son retour, «le feuillage divin du grand chêne de Zeus » ${ }^{25}$. La Toison d'Or, que Jason et ses Argonautes partent conquérir, est suspendue à un chêne qui a, lui aussi, valeur de temple. De la même façon, la forêt de Brocéliande est identifiée à un sanctuaire, à un véritable temple naturel et vivant. Tous ces hauts lieux sylvestres de l'Antiquité, tant païens que monothéistes, ont comme point commun la sacralisation d'un arbre incarnant le sanctuaire où le divin est à même de se révéler sous quelque forme que ce soit. L'arbre, voire la forêt entière, sont alors vénérés par l'homme 
puisqu'ils renferment en eux une part de sacré qui le dépasse et auquel il doit obéissance et respect. C'est ainsi qu'il incarne à la fois le nourricier terrestre et spirituel, un sanctuaire de paix et de méditation comme il en est fait mention à diverses reprises chez Appelfeld. Ainsi sur la colline où réside Gottfried ${ }^{26}$, les arbres tiennent une place prépondérante :

J'ai élu domicile dans cette petite synagogue. La plupart du temps, j'y suis seul, entre les murs et les arbres [...] Par les fenêtres, le regard s'échappe au loin, vers les cimes des montagnes; tout autour, ce ne sont que feuillage chantant, musique et bruissement, brise accrochée aux rideaux ${ }^{27}$.

Les arbres sont une partie intrinsèque du lieu saint dont Gottfried est le gardien. On a l'impression que sans leur présence et toute la vie qu'elle apporte, ce lieu serait incomplet. Le désir de contemplation qu'il éveille chez l'homme émane à la fois du lieu de prière et des arbres, véritables sanctuaires naturels.

23 Ainsi dans la nouvelle La route de Drovno à Drohobycz $z^{28}$, un groupe de Juifs part en pèlerinage à Drohobycz, ville célèbre pour son tsadik. Après avoir dépeint l'allégresse qui s'empare non seulement des hommes, mais aussi des choses, en l'occurrence des roues de la charrette qui martèlent joyeusement le sol, l'auteur fait, au sens propre, une halte pour célébrer l'instant sacré où le père de l'enfant va rompre le pain : «Entre Drovno et Drohobycz, se dresse un arbre. Assurément il y est encore jusqu'à ce jour. En été, à la tombée de la nuit, il ressemble à une forêt où tous les oiseaux sont nichés, où tous les nigunim se réjouissent, et une vapeur légère monte de la rivière. Mon père découpa alors la halla sur une nappe brodée à même la terre ${ }^{29}$. Premier signe de sainteté : l'hospitalité biblique. L'arbre, telle une forêt bienfaisante, accueille les voyageurs sous son feuillage. Autre signe de la sainteté du lieu, il réjouit l'âme du chant joyeux des oiseaux, un nigun d'origine divine, symbole du lien profond qui unit cette petite communauté de Juifs à leur tradition. L'arbre sert également de sanctuaire puisque c'est sous son feuillage que le pain est rompu. Dans le judaïsme, la consommation du pain est toujours précédée d'une bénédiction où l'on rend grâce à Dieu « qui fait sortir le pain de la terre ». Or, c'est précisément ce qui semble se passer au sens propre : en effet, la halla, qui est à l'origine le pain consacré à Dieu, est posée sur le sol comme si elle venait de sortir de terre.

24 L'arbre-sanctuaire est donc un arbre-gardien, à la fois protecteur et réceptacle de la tradition. Il annonce la grande forêt qui va servir de refuge aux fugitifs pourchassés pendant la Shoah. Ainsi dans la nouvelle Trois $^{30}$ qui ouvre le tout premier recueil d'Appelfeld paru en 1962, Fumée, trois hommes viennent de s'échapper d'un camp. Ils se retrouvent errants dans la campagne et, dès la première page, l'auteur précise que "l'enchevêtrement de la forêt leur offrit un abri »"

\section{La forêt, un lieu de métamorphose}

25 Mais la forêt apparaît aussi comme un lieu de métamorphose où les hommes se confondent peu à peu au milieu sylvestre qui leur sert de refuge, ils perdent alors de leur humanité et leurs réactions se font animales. Pour échapper à la persécution, le personnage de La fuite ${ }^{32}$ a pris l'aspect d'un non-Juif. Il passe les nuits d'été dans la forêt :

Il avançait lentement sur son cheval, bercé comme par une sorte de courant.

Parfois, il faisait une halte, le cheval à ses côtés, les voix de la forêt coulaient à ses 
oreilles. Il se sentait chez lui dans cette forêt, comme s'il n'avait jamais entendu parler de brigands, ni de loups, ni d'ours ${ }^{33}$. souligne la force de cette fusion au sein de la forêt. C'est par ailleurs le seul endroit où le personnage se sent «chez lui ». Mais bien que cette métamorphose lui sauve la vie, l'homme se sent dépossédé à nouveau d'une partie de son être une nuit où il rencontre dans la forêt des fugitifs juifs. Ceux-ci ont beaucoup de mal à le reconnaître pour l'un des leurs tant il ressemble à présent en tous points à un non-Juif, rien ne subsiste en lui de ce qu'il a été auparavant. Il éprouve alors un sentiment d'étrangeté absolue pour cette forêt dont il était jusque-là si proche :

Cette nuit-là, la lune éclairait la forêt. Le cheval avançait lentement, en étroite union avec le souffle froid de la forêt. [...] Lui sentait que, faute d'attention, il venait d'être amputé encore de l'un de ses membres ${ }^{34}$.

À l'intérieur de ce cercle magique ou démoniaque qu'est la forêt, les fugitifs qui se terrent au fond des bois sont parfois obligés pour survivre d'adopter un mode de vie animal. Il s'agit alors pour les personnages de revêtir une seconde peau. Dans la nouvelle La métamorphose ${ }^{35}$, Appelfeld montre comment un couple juif pourchassé se fond peu à peu dans la forêt elle-même :

Ce n'est pas vraiment un de mes gestes, se dit-il en ricanant. Les changements avaient été tellement rapides, comme la chute des feuilles; même ce qui restait n'était pas de lui. Une autre peau avait poussé sur son visage, ses mains étaient velues. Elle aussi, elle avait changé en même temps que lui, dans une secrète connivence. [...] Pendant tout l'hiver [les paysans] les avaient pourchassés. Au printemps, ils savaient déjà nager, grimper aux arbres, se blottir dans une caverne, ou, comme les caméléons, se pétrifier contre des pierres ${ }^{36}$.

Ayant réussi à survivre pendant l'hiver, les personnages sont capables, le printemps venu, d'être à l'unisson avec la vie de la forêt et de se couler, en cas de besoin, dans une forme animale, végétale ou même minérale. Mais cette bienveillance de la forêt s'avère à nouveau être un leurre, car les personnages sont en même temps dépossédés de leurs racines, de leur identité et de leur langue. À l'instar du diable qui offre à Faust la jeunesse éternelle en échange de son âme, la forêt laisse la vie sauve aux fugitifs à condition qu'ils se dépouillent de leur essence même.

29 Le même phénomène touche le personnage de La fuite qui subit, lui aussi, une métamorphose totale. Sa langue - le yiddish - lui fait défaut au point que même lors d'une rencontre fortuite avec des Juifs, il est incapable de s'exprimer dans sa langue maternelle :

Il voulait leur parler en langue juive et leur parla dans la langue des non-Juifs. Pour finir, il se mit à jurer comme le font les paysans. « Mais le cheval, vous ne le voulez pas? C'est pour sûr un bon cheval !", fit-il, à la manière des ivrognes qui proposent leur femme pour la nuit ${ }^{37}$.

30 Ainsi, en même temps que le langage, ce sont les souvenirs qui disparaissent. La tradition juive s'estompe lentement et avec elle, les critères moraux qui servaient de repère aux deux personnages de La métamorphose. La femme commence à fréquenter l'église le dimanche. Il se met à la battre, imitant en cela le comportement des nonJuifs. Ils oublient tous deux leur langue maternelle, le yiddish. Puis la femme ne résiste pas à l'appel des fermiers du village et s'enfuit avec eux. L'homme est laissé à lui-même, le corps pris dans les premières neiges. 
31 Le «cercle de la métamorphose ${ }^{38}$ aura donc eu raison de l'homme et la forêt a le dernier mot: symboliquement la mort de l'homme montre bien que ses racines juives demeurent à jamais enfouies et oubliées sous les glaces de l'hiver.

\section{Les langages de la steppe glacée}

32 S'il arrive dans les nouvelles d'Appelfeld que les personnages oublient leurs racines, volontairement ou non, l'oubli s'exprime, l'oubli « se parle ». Il vit même, bien que figé dans un paysage de steppes glacées. C'est de ces profondeurs gelées que l'écrivain s'efforce de le tirer et de lui insuffler mémoire et vitalité.

33 La neige peut servir de révélateur à un autre paysage. Ainsi Madame Traum ${ }^{39}$, revenant dans sa ville natale en Allemagne, retrouve sous le lourd manteau de l'hiver les images printanières chères à son enfance. Mais celles-ci se détériorent rapidement au contact d'autres souvenirs et les flocons tout d'abord bienveillants se révèlent être des plus hostiles. La neige est non seulement capable d'éveiller la nostalgie des étendues immaculées, mais elle a également le pouvoir de dévoiler des paysages enfouis, des visions d'autres saisons. Lorsque Madame Traum regarde tomber les flocons, le regard de sa mémoire parvient à capter la colline verdoyante de son enfance qui surgit au milieu du tourbillon neigeux.

34 Or très vite, les allusions inquiétantes se multiplient : des « corbeaux étrangers » ont élu domicile sur les arbres du parc. La couleur noire tranche avec la pureté immaculée tout alentour. Le terme "étrangers " évoque en filigrane la peur de l'inconnu, peur aussitôt justifiée par le hurlement du train. La vision de la fumée achève de transformer ce rêve éveillé en véritable cauchemar qui ramène l'héroïne aux angoisses qu'elle tente vainement de refouler. Face à l'agressivité de l'hiver, elle préfère renoncer à l'évocation de souvenirs qui lui sont chers et se recroqueville dans les seuls gestes de la vie courante.

35 De l'idéalisation du paysage enneigé de l'enfance au souvenir douloureux, vite réfréné, voire étouffé, Appelfeld décrit aussi le processus d'amnésie causé par le froid et la glace. La mémoire s'atrophie et est prise par le gel qui fige et pétrifie les souvenirs de « là-bas ».

36 La steppe enneigée représente simultanément un lieu de mort ou de salut. Dans la nouvelle Sibérie $e^{40}$, pendant la guerre, des déportés sont affectés au transport du charbon vers le front. Mais le train est miraculeusement bloqué par la neige. Celle-ci, secondée par le vent, envahit l'espace entier; le ciel et la terre se rejoignent dans une communion neigeuse. Les éléments du froid semblent se liguer pour semer le désordre et de cette façon venir en aide aux opprimés et aux oubliés. Les détenus, évacués vers un cabanon, profitent de cette halte providentielle pour faire provision de chaleur et de repos. De la sorte, la neige "donne naissance " à la chaleur puisque les prisonniers auront tout loisir d'utiliser le charbon destiné au front pour se chauffer eux-mêmes.

37 La neige va même jusqu'à devenir rédemptrice et sauver des vies humaines de la terrible conscription. La nouvelle La mort de l'intercession ${ }^{41}$ relate les efforts désespérés du représentant d'une petite communauté juive au XIX ${ }^{\mathrm{e}}$ siècle pour éviter à la fine fleur des garçons du village d'être réquisitionnés dans l'armée tsariste. En fin de compte, grâce à la tempête de neige, le pire sera peut-être épargné aux jeunes gens. 
38 La steppe enneigée présente donc ce double visage de Janus, aspect déjà observé pour l'eau et la forêt. La neige est aussi bien lieu de mort que source de vie.

\section{Quand la glace devient flamme : de l'oubli à la mémoire}

39 Si la steppe glacée symbolise l'oubli, la flamme, elle, représente souvent chez Appelfeld le réveil de la mémoire. C'est ainsi que les récits de voyageurs de commerce ${ }^{42}$ véhiculent au milieu d'un paysage enneigé quelques bribes de chaleur susceptibles de ranimer une mémoire endormie.

40 Dès l'ouverture de la nouvelle Gel sur la terre ${ }^{43}$, Appefeld en donne la tonalité : au cœur de l'hiver les éléments se sont ligués pour faire disparaître tout souvenir de ce qui fut. La neige a recouvert les mémoires de son épais manteau, quant aux vents, allégés de leur fardeau, ils soufflent désormais dans le vide et ne produisent que du néant. Les derniers signes de vie humaine sont impitoyablement oblitérés comme pour ne laisser aucune marque, si ténue soit-elle. Mais une petite lumière veille dans la nuit: l'hôtelpension où chaque année, à pareille époque, le voyageur de commerce trouve refuge. L'aubergiste du lieu incarne la mémoire vive de toute la contrée : lui-même et toute sa maison recèlent des myriades de souvenirs mi-réels, mi-imaginaires sur les familles juives de toute la région. Le temps semble vouloir les épargner afin que la tradition se perpétue et que le souvenir des racines familiales ne s'éteigne pas. C'est bien entendu au coin $\mathrm{du}$ feu que sont relatés les récits, l'hôtelier faisant figure de grand-père racontant aux plus jeunes l'histoire de leurs familles. La proximité de la flamme a ici une importance majeure: elle ravive une maison, des personnages disparus dans la neige et relie le narrateur à sa mémoire, renouvelant ainsi la symbiose originelle entre les deux.

41 C'est à nouveau dans la nouvelle Sibérie que l'on trouve les rapprochements les plus contrastés entre glace et feu. Grâce à une tempête de neige, les détenus, installés bien au chaud dans un cabanon, peuvent tout à loisir contempler les circonvolutions de la neige à l'extérieur :

La porte avait gelé sous les amoncellements de neige. Le cabanon était bien protégé. Qu'il faisait bon s'étirer, sentir la neige vivante sous le plancher, se tenir tout contre le flot du vent, partir loin d'ici léché par des langues de feu, se laisser entrainer dans ce cercle encore et encore pour finalement poser l'ancre à nouveau sur ce plancher [...]. Quelqu'un avait fait fondre la glace de la lucarne et en avait ôté la cloison. La neige striait l'espace, une neige pleine. Semblables à des pétales, les flocons s'ouvraient et fondaient silencieusement ${ }^{44}$.

42 Ainsi le froid engendre le chaud et c'est au point que les tourbillons du vent et de la neige évoquent dans l'imaginaire des prisonniers des «langues de flamme». La symbiose entre le feu et la glace atteint ici son apogée.

\section{Quand le soleil prend la couleur du gel}

Il arrive aussi que des personnages qui ont su se protéger des morsures de la mémoire soient inexorablement attirés vers l'un de leurs semblables. Ils font resurgir ce qu'ils croyaient à jamais éteint, réactivant de la sorte une douleur enfouie. Le soleil qui semblait veiller sur eux se trouve à son tour "contaminé » par cette évocation et prend, lui aussi, les couleurs du gel. 
44 Dans Soleil d'hiver ${ }^{45}$, Appelfeld entrelace finement divers motifs du gel, mais s'attarde surtout, en point d'orgue, sur cette emprise progressive du gel sur le soleil. L'existence paisible d'un commerçant célibataire est peu à peu troublée par Rosa, serveuse de restaurant. Le passé de Fromm, les années de guerre et d'avant-guerre, semblent dormir en lui sans le tourmenter outre mesure. Tout dans son existence indique qu'il ne souhaite pas être dérangé et surtout pas par ses propres souvenirs, prenant bien garde de ne pas les réveiller. Or il s'avère que tous deux, Fromm et Rosa, sont originaires de bourgades proches l'une de l'autre, traversées par le même fleuve, le Prut. Et la magie de la réminiscence opère : Fromm commence à se souvenir.

45 Grâce à la sensation aiguë de froid et à la vision d'une nuit lumineuse, Fromm réussit à s'extirper de sa gangue habituelle et se retrouve physiquement dans le paysage de son enfance. Ce retour vers lui-même, vers un passé si soigneusement enseveli, emplit Fromm d'un "soleil d'hiver", métaphore d'un bonheur intense qui accompagne le couple. Mais on ne peut évoquer impunément la mémoire enfouie. Bien que les personnages tentent se prémunir contre la douleur qui les submerge, l'évocation de cette vie disparue ne peut les laisser indemnes.

46 Sur le plan métaphorique, le soleil d'hiver, symbole du bonheur de Fromm et de Rosa, change peu à peu de couleur. Il passe tout d'abord par les tons du bleu, couleur froide s'il en est, pour prendre finalement la teinte du gel lui-même, une teinte de métal argenté. Insensiblement, la lumière de l'hiver prend le dessus. Au lieu du «soleil d'hiver » qui fait contrepoint avec le gel, c'est un "soleil froid et argenté » qui règne désormais sur la ville et principalement sur les esprits. L'empreinte de ce gel-argent est partout, la lumière est hivernale, le froid attaque la transparence des vitres, rendant impossible une vision directe de la réalité. La lumière du soleil ainsi opacifiée par la teinte argentée devient en même temps le symbole de la séparation et de l'absence de communication: en tant que muraille, elle ne laisse même plus les voix parvenir jusqu'à elle. L'incommunicabilité, le sentiment d'étrangeté et d'agression par rapport à la réalité extérieure, la sensation de dispersion et d'éparpillement, toutes ces composantes mènent progressivement les deux personnages à une rupture totale d'avec l'univers qui les entoure.

47 La nouvelle s'achève sur une sensation de "vide infini ", très loin du réel, et dans lequel Fromm semble s'enfoncer pour l'éternité. Rosa et Fromm ont cru pouvoir ensemble retourner à leur vie passée, à leurs racines enfouies. Mais leur innocente et émouvante complicité se heurte à leur douleur intérieure, elle-même sous le joug du gel porteur d'oubli. Celui-ci aura le dernier mot, entachant leur univers ensoleillé de sa couleur argentée, métallique, couleur de mort.

48 Ces trois langages de la nature, l'eau, la forêt et la glace, sont fondamentaux dans toute l'œuvre d'Aharon Appelfeld qui les a modelés et façonnés dès ses tout premiers écrits. À travers eux il tisse un paysage intérieur de symboles doués d'une vie propre qui transcendent l'état de langage pour incarner des êtres de chair et de sang, réels et palpables à travers les personnages qui les évoquent. Éléments naturels, langages, symboles et personnages se rejoignent ainsi en une surprenante osmose où les voix entrelacées se répondent, telles des correspondances baudelairiennes, dans une tonalité et une harmonie toujours justes. 


\section{BIBLIOGRAPHIE}

APPELFELD, Aharon, recueils de nouvelles

- ‘Ashan (« Fumée »), Jérusalem, Achshav, 1962.

- Ba-gay ha-poreh (« Dans le vallon fertile »), Tel Aviv, Schocken, 1963.

- Kfor 'al ha-arets (« Gel sur la terre »), Ramat Gan, Massada, 1965.

- Be-qomat ha-qarqa“ (« Au rez-de-chaussée »), Tel Aviv, Daga, 1968.

- Adnei ha-nahar (« Les rives du fleuve »), Tel Aviv, Hakibbutz Hameuchad, 1971.

- Ke-me'a 'edim (« Comme cent témoins »), Tel Aviv, Hakibbutz Hameuchad, 1975.

HOMÈRE, L'Odyssée, Paris, Gallimard, coll. « La Pléiade », 1955.

RAMRAS-RAUCH, Gila, The Holocaust and Beyond, Indianapolis, Indiana University Press, 1996.

RATTOK, Lily, Bayit 'al blima (« La poétique de la prose chez Aharon Appelfeld »), Tel Aviv, Heker, 1989.

\section{NOTES}

1. Son premier recueil, 'Ashan, a été publié en 1962. Pour les traductions de ses nouvelles en français : Kitty, in Anthologie de la prose israélienne, Paris, Albin Michel, 1980, p.121-136, trad. Françoise Rameau-Le Davay; Regina, Les Nouveaux Cahiers n 85, 1986, p. 59-63, trad. Arlette Pierrot; Le dernier refuge, in Anthologie de nouvelles israéliennes contemporaines, Paris, Gallimard, 1998, p. 37-49, trad. Gisèle Sapiro ; Métamorphose et Une histoire d'amour, Tsafon n ${ }^{\circ} 37,1999$, p. 159-171, trad. Arlette Pierrot.

2. Quelques nouvelles d'Aharon Appelfeld publiées dans des revues littéraires :

Ha-nissayon («La tentative »), Moznaim $\mathrm{n}^{\circ} 30$, avril-mai 1970, p. 363-365; Tsel tsohorayim (« L'ombre de midi »), 'Akhshav n ${ }^{\circ}$ 25-28, printemps 1973, p. 173-177 ; Ahava me'uheret (« Un amour tardif »), Iton $77 \mathrm{n}^{\circ} 18,1979$, p. 4-5; Ha-minyan ha-'avud (« Le minyan perdu »), 'Aley siyah $\mathrm{n}^{\circ} 23$, 1985, p. 129-137; Be-shuley ha-'ir shelanu ("À la lisière de notre ville »), Qeshet ha-hadasha ${ }^{\circ} 1$, automne 2002, p. 28-42.

3. Aharon Appelfeld (1965), Pgisha, in Kfor 'al ha-arets ("Gel sur la terre »), Ramat Gan, Massada, p. 123-128.

4. A. Appelfeld (1968), Rushka, in Be-qomat ha-qarqa“ («Au rez-de-chaussée »), Tel Aviv, Daga, p. 84-96.

5. A. Appelfeld (1968), Ha-hishtannut, in Au rez-de-chaussée, op. cit. (p. 55-62). Traduction d'Arlette Pierrot, Tsafon $\mathrm{n}^{\circ} 37,1999$, p. 160.

6. Ibid., p. 160.

7. A. Appelfeld (1975), Ke-'ishon ha-'ayin, in Ke-me'a 'edim ("Comme cent témoins »), Tel Aviv, Hakibbutz Hameuchad, p. 195-270.

8. Ibid., p. 205. Toutes les traductions, sauf précision contraire, sont de l'auteur de l'article.

9. Ibid., p. 209-210.

10. A. Appelfeld (1963), 'Al-yad ha-hof, in Ba-gay ha-poreh (« Dans le vallon fertile »), Tel Aviv, Schocken, p. 116-134.

11. C'est nous qui soulignons.

12. A. Appelfeld (1963), Sur le rivage, op. cit. p. 116. 
13. Ibid.

14. A. Appelfeld (1971), Be-Hanoucca 1946, in Adnei ha-nahar ("Les rives du fleuve »), Tel Aviv, Hakibbutz Hameuchad, p. 103-107.

15. Ibid., p. 103.

16. A. Appelfeld (1965), Be-iyyei Saint George, in Gel sur la terre, op. cit. p. 101-122.

17. Gila Ramras-Rauch (1994), The Holocaust and Beyond, Indianapolis, Indiana University Press, p. 68.

18. Lily Rattok (1989), Bayit 'al blima (« La poétique de la prose chez Aharon Appelfeld »), Tel Aviv, Heker, p. 136.

19. Ibid., p. 127.

20. A. Appelfeld (1963), Ba-gay ha-poreh, in Dans le vallon fertile, op. cit., p. 108-115.

21. Ibid., p. 108-109.

22. A. Appelfeld (1962), Le’aț, in 'Ashan (« Fumée »), Jérusalem, Achshav, p. 79-85.

23. Ibid., p. 79-80.

24. Genèse XII, 6-7 ; XIII, 14-18.

25. Homère, L'Odyssée, XIV, 327 ; XIX, 296-298.

26. A. Appelfeld (1965), Mi-merom ha-dumiyya («Du haut du silence »), in Gel sur la terre, op. cit., p. $90-94$.

27. Ibid., p. 90-91.

28. A. Appelfeld, (1965), Ha-derekh beyn Drovno le-Drohobycz, in Gel sur la terre, op. cit., p. 7-13.

29. Ibid., p. 7.

30. A. Appelfeld (1962), Shlosha, in Fumée, op. cit., p. 7-13.

31. Ibid., p. 7.

32. A. Appelfeld (1968), Ha-beriha, in Au rez-de-chaussée, op. cit., p. 5-20.

33. Ibid., p. 9.

34. Ibid., p. 19-20.

35. A. Appelfeld (1968), La métamorphose, op. cit.

36. Ibid., p. 55.

37. A. Appelfeld, La fuite, op. cit., p. 19.

38. A. Appelfeld, La métamorphose, op. cit., p. 160.

39. A. Appelfeld (1962), Pitsuyim ("Indemnités »), in Fumée, op. cit., p. 17-48.

40. A. Appelfeld (1963), Sibir, in Dans le vallon fertile, op. cit., p. 83-100.

41. A. Appelfeld (1965), Motah shel ha-shtadlanut, in Gel sur la terre, op. cit., p. 23-28.

42. A. Appelfeld (1965), Mi-sodot ha-mishar ha-za'ir («Les secrets du petit commerce »), p. 37-43; Kfor 'al ha-arets (« Gel sur la terre»), p. 86-89; (1968), Ba-derekh (« En route»), p. 63-68, in Gel sur la terre.

43. A. Appelfeld (1965). Kfor 'al ha-arets, in Gel sur la terre, op. cit., p. 86-89.

44. A. Appelfeld, Sibérie, op. cit., p. 95.

45. A. Appelfeld (1971), Shemesh shel horef, in Les rives du fleuve, op. cit., p. 151-157.

\section{RÉSUMÉS}

Avant d'entamer un fructueux parcours de romancier, Aharon Appelfeld a commencé sa carrière en tant que nouvelliste. Il a publié cinq recueils de nouvelles entre 1962 et 1971, sans 
compter de multiples textes qui ont vu le jour dans des revues littéraires.

C'est dans la nature et ses éléments multiples qu'Aharon Appelfeld puise les langages qui lui permettent de forger une symbolique où la polysémie n'a d'égal que les variations musicales de la langue hébraïque. Tour à tour, l'eau, la forêt, la steppe glacée ou la brûlure du soleil, la flore protectrice ou nocive ainsi que la faune domestique ou sauvage deviennent des moyens d'expression, de véritables langages où affleurent sensations, souvenirs et états d'âme. La nature incarne ainsi la muse inspiratrice d'une palette de nouveaux symboles créés par l'artiste.

Before advancing onto the profitable, fruitful path of a novelist, Aharon Appelfeld began his career as a short story writer. Between 1962 and 1971, he wrote no less than five collections without including the numerous texts published in literary magazines.

It is nature and its multiple elements that inspired his language and allowed him to create a symbolism where polysemy can only compare to the musical variations of the Hebrew language. In turn, water, the forest, the frozen tundra or the burning of the sun, the protective or harmful flora as well as the domestic or wild fauna, became modes of expression inspired by genuine languages in which sensations, memories and states of mind become manifest. Nature embodies the inspiring muse of a palette of new symbols created by the artist.

לפני שהחל בכתיבת רומנים, פרסם אהרון אפלפלד חמישה קובצי סיפורים מלבד טקסטים נוספים

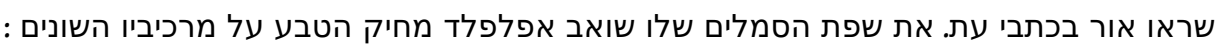

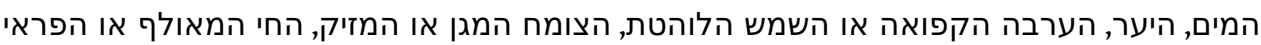

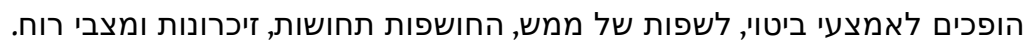

\section{INDEX}

Thèmes : littérature

Keywords : Appelfeld's short stories, nature, water, forests, memory, changes, literature, Appelfeld Aharon (1932-)

\section{מילות מפתח}

טבע, מים, יער, ערבה קפואה, זיכרון, שכחה, השתנות:

Mots-clés : langages, nature, eau, forêt, steppe glacée, sanctuaire, métamorphose, mémoire, oubli, Appelfeld Aharon (1932-) 